\title{
CANCELLATION COUNTEREXAMPLES IN KRULL DIMENSION 1
}

\author{
ROBERT M. GURALNICK, LAWRENCE S. LEVY, AND ROBERT B. WARFIELD, JR.
}

(Communicated by Louis J. Ratliff, Jr.)

\begin{abstract}
We show that direct-sum cancellation can fail for modules in a single genus, over commutative noetherian rings of dimension 1. The example provides an application of noncommutative methods (including "wild representation type") to commutative ring theory.
\end{abstract}

Let $\Lambda$ be a module-finite algebra over a commutative ring $R$. We say that a $\Lambda$-module $N$ is in the genus of $M$, and write $N \in \operatorname{gen}(M)$, if $N_{\mathrm{m}} \cong M_{\mathrm{m}}$ (as $\Lambda_{\mathfrak{m}}$-modules) for all maximal ideals $m$ of $R$. (In this paper "module" means "finitely generated left module", unless the contrary is stated.) We say that cancellation holds in $\operatorname{gen}(M)$ if

$$
L \oplus X \cong N \oplus X \Rightarrow L \cong N \quad \text { when } L, N, X \in \operatorname{gen}(M) .
$$

In [GL '90] it was shown that, if $\Lambda$ is a commutative noetherian ring of Krull dimension 1 without nilpotent elements, then cancellation holds in every genus of $\Lambda$-modules. We give an example showing that this can fail if $\Lambda$ has nilpotent elements. In our example, the abelian groups $(\Lambda,+)$ and $(M,+)$ are free of finite rank; and the example shows that such failure of cancellation is quite common in this situation. The example makes use of the notion of wild representation type to transfer a noncommutative example of Swan (involving totally definite quaternion algebras) to this commutative situation.

1. Notation. Let $\Lambda$ be a commutative $Z$-algebra such that the abelian group $(\Lambda,+)$ is free of finite rank. Suppose that some maximal ideal $\mathfrak{m}$ of the artinian ring $A=\mathbf{Q} \Lambda$ requires at least three generators and $A / \mathfrak{m} \cong \mathbf{Q}$. Such a $\Lambda$ has Krull dimension 1 because it is integral over $\mathbf{Z}$.

For example, we can take $\Lambda=\mathbf{Z}[x, y, z] /\langle x, y, z\rangle^{2}$.

2. Theorem. There is a $\Lambda$-module $M$ such that $(M,+)$ is free of finite rank and cancellation does not hold in $\operatorname{gen}(M)$.

Proof. The $\mathbf{Q}$-algebra $A=\mathbf{Q} \Lambda$ has wild representation type, by which we mean that there is a representation equivalence $\Phi: \mathscr{C} \rightarrow \mathscr{F}$, from a full subcategory

Received by the editors April 24, 1989 and, in revised form, August 8, 1989.

1980 Mathematics Subject Classification (1985 Revision). Primary 13C05, 16A64.

The authors were partially supported by NSF grants DMS 8901492, 8620430, and 8944436. 
$\mathscr{C}$ of the category of left $A$-modules, to the category $\mathscr{F}$ of all left modules over the free algebra $\mathbf{Q}\langle s, t\rangle$ in two noncommuting indeterminates.

The definition of representation equivalence $\Phi: \mathscr{C} \rightarrow \mathscr{F}$ is not completely standard. We define it to be an additive functor $\Phi$ such that: (i) Every isomorphism class in $\mathscr{F}$ occurs in the image of $\Phi$; (ii) $\Phi(M) \cong \Phi(N)$ if and only if $M \cong N$; and (iii) $\Phi$ is a surjection on hom groups. We will use the fact that, in this particular situation, $\Phi$ can be chosen to have the following additional property: (iv) $M$ is finite-dimensional if $\Phi(M)$ is.

The existence of $\Phi$ follows from a slight modification of [W'78, Theorem 1], whose proof we sketch in Lemma 3 below. The existence of $\Phi$ might also follow from an earlier theorem of Drozd [D '72], but we do not know whether Drozd's functor has property (iii) in our definition of "representation equivalence", which will be needed below.

Every finite-dimensional Q-algebra $H$ can occur as the endomorphism ring of some finite-dimensional $\mathbf{Q}\langle s, t\rangle$-module. (This is true for any field in place of Q.) [B'74, p. 134; Ga '75, pp. 148-149]

Let $H$ be a Q-algebra such that direct-sum cancellation fails for projective modules $\Gamma$-modules in $\operatorname{gen}(\Gamma)$ over some maximal Z-order $\Gamma$ in $H$. Such an $H$ (a totally definite quaternion algebra) exists by [S '62]. Then we have $H=E(V)$, the endomorphism ring of some finite-dimensional $\mathbf{Q}\langle s, t\rangle$-module $V$. Our representation equivalence $\Phi$ yields a finite-dimensional $A$-module $M^{*}$ such that $V \cong \Phi\left(M^{*}\right)$.

Let $M$ be any (necessarily $\mathrm{Z}$-free) finitely generated $\Lambda$-submodule of $M^{*}$ such that $\mathbf{Q} M=M^{*}$. We claim that cancellation fails in the genus of $M$.

First of all, $V$ is an indecomposable $\mathbf{Q}\langle s, t\rangle$-module, since $E(V)$ is a division ring. Since $\Phi$ is a representation equivalence, $M^{*}$ is therefore an indecomposable $A$-module. Since $\Phi$ is a surjection on hom groups, we have $\Phi\left(E\left(M^{*}\right)\right)=H$, and this is a homomorphism of $\mathbf{Q}$-algebras (since $\Phi$ is an additive functor). Moreover, since the $A$-module $M^{*}$ is indecomposable and of finite length, its endomorphism ring is a local ring, so we have

$$
\operatorname{ker}\left(\Phi: E\left(M^{*}\right) \rightarrow H\right)=\operatorname{rad} E\left(M^{*}\right)
$$

Now consider $E(M)$. We have $\mathbf{Q} \cdot E(M)=E(\mathbf{Q} M)=E\left(M^{*}\right)$. Since $\Phi$ is a Q-algebra homomorphism on $E\left(M^{*}\right)$, we see that $\Phi(E(M))$ is a Z-order in $H$. Since cancellation fails in $\operatorname{gen}(\Gamma)$ for some maximal Z-order $\Gamma$ in $H$, it fails in $\operatorname{gen}(\Lambda)$ for every Z-order $\Lambda$ in $H$ (by Lemma 4, below). Therefore cancellation fails for $\Phi(E(M))$-modules in $\operatorname{gen}(\Phi(E(M)))$.

By (2.1), and the fact that $\Phi(E(M))$ is an integral domain, the kernel of $E(M) \rightarrow \Phi(E(M))$ equals the nilradical of $E(M)$. So, by the proof of [G '84, 3.5], failure of cancellation in $\operatorname{gen}(\Phi(E(M)))$ lifts to failure in $\operatorname{gen}(E(M))$ and then to $\operatorname{gen}(M)$, as desired. 
3. Lemma. Let $A$ be a commutative artinian ring with a maximal ideal $\mathfrak{m}$ that requires at least three generators, and let $k=A / \mathfrak{m}$. Then there is a representation equivalence $\Phi$ from a full subcategory of $\bmod -A$ to the category of all left modules over the free noncommutative $k$-algebra $k\langle s, t\rangle$. Moreover, $M$ has finite length if $\Phi(M)$ is finite-dimensional.

Proof. If $H$ is an ideal of $A$, then every $A / H$-module is also an $A$-module. So it suffices to prove the lemma with $A / H$ in place of $A$, for some convenient $H$. We use this to achieve some simplifications. Since $A$ is artinian and commutative, it is a direct product of local rings. So we can assume that $A$ is local with maximal ideal $\mathfrak{m}$. After replacing $A$ by $A / \mathfrak{m}^{2}$ we can suppose that $\mathfrak{m}^{2}=0$. The $k$-dimension of $\mathfrak{m}$ is now the minimal number of generators of $\mathfrak{m}$. So after passing to another homomorphic image of $\mathfrak{m}$ we can suppose that this dimension is exactly 3 .

Let $a, b, c$ be a $k$-basis of $\mathfrak{m}$, hence a set of generators for the ideal $\mathfrak{m}$. If $M$ is any $A$-module, multiplication by $a, b, c$, respectively, induces $k$ linear transformations $\alpha, \beta, \gamma$ from $M / \mathfrak{m} M$ to $\mathfrak{m} M$. We say that $M$ is a-translatable if $\alpha$ is an isomorphism.

If $M$ is $a$-translatable, we define linear transformations ("translations") $s, t: M / \mathfrak{m} M \rightarrow M / \mathfrak{m} M$ by $s=\alpha^{-1} \beta$ and $t=\alpha^{-1} \gamma$ ( $\beta$ or $\gamma$ first, then $\left.\alpha^{-1}\right)$. This gives $M / \mathfrak{m} M$ a natural left $k\langle s, t\rangle$-module structure. If $M$ and $N$ are $a$-translatable $R$-modules, and $f: M \rightarrow N$ is a homomorphism, then $f$ induces a $k\langle s, t\rangle$-module homomorphism $M / \mathfrak{m} M \rightarrow N / \mathfrak{m} N$. We therefore have a functor $\Phi$ from the category of $a$-translatable $A$-modules to the category of left $k\langle s, t\rangle$-modules.

In the original theorem in [W $\left.{ }^{7} 78\right], \mathfrak{m}$ had $k$-dimension 2 , and the present role of $k\langle s, t\rangle$ was played by the commutative polynomial ring $k[t]$.

The proof of the present lemma is identical to that of [W 78 ], with the following change. Every submodule $K$ of a free $k[t]$-module $F$ is again free, because $k[t]$ is a principal ideal domain. (This is used on p. 453, first full paragraph.) Although $k\langle s, t\rangle$ is not a principal ideal domain, it is a free ideal ring, and over any such ring, submodules of free modules are free $\left[C^{\prime} 71, p\right.$. 80].

4. Lemma. Suppose that cancellation fails in $\operatorname{gen}(\Gamma)$, for some maximal $\mathbf{Z}$ order $\Gamma$ is some $\mathbf{Q}$-division algebra $H$. Then it fails in $\operatorname{gen}(\Lambda)$ for every $\mathbf{Z}$-order $\Lambda$ in $H$.

Proof. Since all maximal orders in $H$ are Morita equivalent to each other [R '75, (2.17)], cancellation in gen $\left(\Gamma^{\prime}\right)$ fails over all maximal orders $\Gamma^{\prime}$ in $H$. Since, in Q-division algebras, every order is contained in a maximal order, it suffices to show that this failure of cancellation passes down from maximal orders to all orders contained in them. This is a theorem of Fröhlich [F'75], and is also a special case of [GL ' $89,5.4]$. 


\section{REFERENCES}

[B '74] S. Brenner, Decomposition properties of some small diagrams of modules, Symposia Mathematica 13 (1974), 127-141.

[C '71] P. M. Cohn, Free rings and their relations, Academic Press, London 1971.

[D '72] Yu. A. Drozd, Representations of commutative algebras (Russian), Funkstional'nyi Analiz i Ego Prilozheniya 6 (1972), 41-43; English Translation in Functional Analysis and its Applications 6 (1972).

[F'72] A. Fröhlich, Locally free modules over arithmetic orders, J. Reine Angew. Math. 274/275 (1975), 112-124.

[Ga '75] P. Gabriel, Representations indecomposable, Seminaire Bourbaki vol. 1973/74, pp. 143169; Lecture Notes in Math., vol. 431, Springer-Verlag, Berlin, 1975.

[G '84] R. M. Guralnick, The genus of a module, J. Algebra 18 (1984), 169-177.

[G1 '90] R. M. Guralnick and L. S. Levy, Cancellation and direct summands in dimension 1, J. Algebra (to appear).

[R '75] I. Reiner, Maximal orders, Academic Press; New York 1975.

[S '62] R. G. Swan, Projective modules and maximal orders, Ann. of Math. (2) 76 (1962), 55-61.

[W '78] R. B. Warfield Jr., Large modules over Artinian rings, Ring Theory, Waterloo 1978, (R. Gordon, ed.), Marcel Dekker, New York 1978, 451-463.

Department of Mathematics, University of Southern California, Los Angeles, CaliFORNIA 90089

Department of Mathematics, University of Wisconsin, Madison, Wisconsin 53706

Department of Mathematics, University of Washington, Seattle, Washington 98195 